\title{
Stochastic Solar Harvesting Characterization for Sustainable Sensor Node Operation
}

Swades De, K. Kaushik and Deepak Mishra

The self-archived preprint version of this journal article is available at Linköping University Institutional Repository (DiVA):

http:/ / urn.kb.se/ resolve?urn=urn:nbn:se:liu:diva-156035

N.B.: When citing this work, cite the original publication.

De, S., Kaushik, K., Mishra, D., (2019), Stochastic Solar Harvesting Characterization for Sustainable Sensor Node Operation, IET Wireless Sensor Systems, , 1-11. https:/ / doi.org/ 10.1049/ ietwss.2018.5009

Original publication available at:

https:// doi.org/ 10.1049/ iet-wss.2018.5009

Copyright: Institution of Engineering and Technology (IET)

http:// www.theiet.org/ 


\title{
Stochastic Solar Harvesting Characterization for Sustainable Sensor Node Operation
}

\author{
K Kaushik, Deepak Mishra, and Swades De
}

\begin{abstract}
Self-sustainability of low power wireless sensor nodes is the need of the hour to realize ubiquitous wireless networks. To address this requirement we investigate the practical feasibility of sustainable green sensor network with solar-powered nodes. We propose simple yet efficient (i) analytical circuit model for solar panel assisted supercapacitor charging and (ii) statistical model for characterizing the solar intensity distribution. Combining these circuit and statistical models, we derive a novel solar charging rate distribution for the solar-powered supercapacitor. To gain analytical insights, we also propose an ideal diode based tight approximation for the practical supercapacitor charging circuit model. The accuracy of these proposed analytical models have been validated by extensive numerical simulations based on the real-world data, i.e., solar intensity profile and solar panel characteristics. The derived solar charging rate distribution is used to investigate the supported sampling rate of the node with different varying number of on-board sensors for a given energy outage probability. Results suggest that for an energy outage probability of 0.1 , at New Delhi, a $40 \mathrm{~F}$ supercapacitor and a 3 W solar panel can support the operation of Waspmote with 6 on-board toxic gas sensors with a sampling rate of 65 samples per day. Further, we use the proposed models to estimate the practical supercapacitor and solar panel sizes required to ensure sustainability of sensor node operation at different geographical locations with varying sensing rate.
\end{abstract}

\section{INTRODUCTION}

Miniaturization of sensors has made wireless sensor network (WSN) technology available for applications such as toxic gas sensing, video surveillance. However, the energy consumption of these sensors often surpasses that of highest energy consuming component (radio) of a traditional wireless sensor node [1]. Energy harvesting has emerged as a potential green solution to address this demand [2]. Among the available ambient sources for harvesting, solar energy has the highest power density and though solar energy is uncontrollable, it can be predicted as a function of location and time [3], [4], [5]. Such a useful energy source, when characterized properly can meet the devices energy demand with minimal hardware resources, i.e., solar panel and supercapacitor sizes.

Although there exists many recent deployments [6], [7] of such WSN with high energy consuming sensors, the feasibility of a sustainable operation of such network with the available resources needs to be evaluated for the deployment location before the actual deployment of nodes. In this work, an air

K Kaushik and S. De are with Department of Electrical Engineering and Bharti School of Telecom, Indian Institute of Technology Delhi, India (email: swadesd@ee.iitd.ac.in)

D. Mishra is with the Communication Systems Division of the Department of Electrical Engineering at the Linköping University, 58183 Linköping, Sweden (email: deepak.mishra@liu.se). pollution monitoring WSN has been considered where the nodes harvest solar energy for sustainable operation.

\section{A. Motivation and Scope}

In order to optimally use the harvested energy for transmission, [8], [9], [10] focus more on developing transmission policies rather than how energy is harvested. The study in [11] considered that the harvested energy is proportional to the solar intensity. However, this assumption is not very practical as the present generation solar energy harvesting nodes use supercapacitors, where the rate of harvesting depends on the residual voltage in the supercapacitor apart from the available solar intensity. A simulation model for charging a supercapacitor using solar panel was developed in [3]. Instead of using practical solar panel models [12], [13], the study in [3] used a simple model, and the charging parameters was found through experiments. So, there is a need to develop an analytical charging model for a solar harvesting sensor node to evaluate its performance directly from the parameters available in the solar panel datasheet, solar intensity distribution at the location where sensor node would be deployed, and the sensor node's energy consumption. Moreover, dimensioning of solar panel and supercapacitor sizes for WSN applications is still missing in the literature.

In this work we address this gap by proposing simple, yet practical, analytical models to accurately characterize the sustainability of a solar energy harvesting WSN node. Though for analysis we consider a periodic data collection application [14] where the sensor nodes are equipped with wake-up receiver for green data communication, the findings are applicable to sustainability studies of any solar harvesting WSNs irrespective of the type of data collection.

\section{B. Contributions}

The key contributions of this work are as follows:

1) A novel distribution model is proposed to characterize the spatio-temporal randomness of solar intensity which can be used at any geographical location on earth.

2) Solar charging rate of supercapacitor is derived for two circuit-level analytical models of solar panel.

3) Solar charging rate distribution and energy outage probability are derived using solar intensity distribution models, which are also validated by simulations.

4) Numerical investigation on the tradeoff between energy outage and sustainable sensing rate is carried out at different places with widely varying solar intensity profiles. 


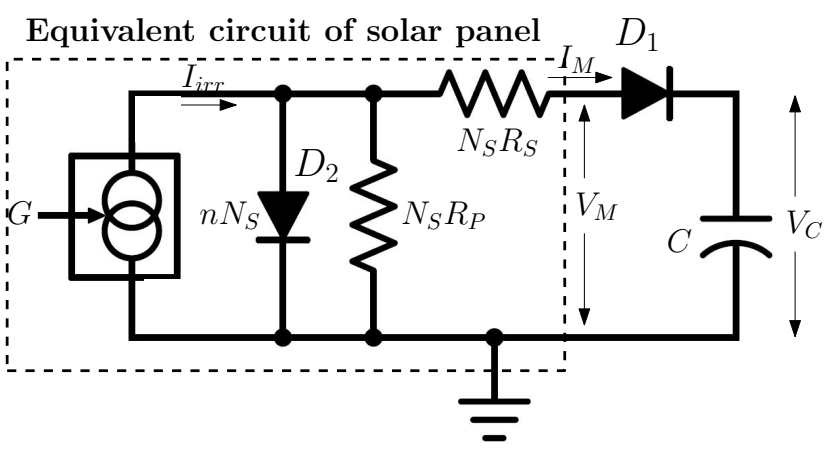

Fig. 1: Circuit for charging a supercapacitor using solar panel.

5) Insights on practical supercapacitor and solar panel sizes required to meet a sustainable rate demand are presented.

\section{PRIOR ART}

\section{A. WSN architectures for solar energy harvesting}

Solar energy harvesting WSN deployments can either be indoors [15] or outdoor [6], where data is typically collected by the base station via multi-hop data transfer. In order to increase the lifetime of WSN, the nodes are grouped into clusters and the the cluster heads aggregate data from its cluster nodes and send it to remote base station. Dynamic clustering via LEACH protocol [16] helps in further increasing the lifetime. However, such protocols may not be needed in solar energy harvesting WSNs as they harvest sufficient energy. Recently, there have been proposals to use heterogeneous nodes where the cluster heads harvest solar energy while the nodes in the cluster RF harvest energy either sent by cluster heads or by a mobile entity [17]. However, all the high energy consuming nodes of air pollution monitoring WSN require solar energy to support its high energy consumption. In this work we consider that all nodes are equipped with solar panels and a mobile entity collects data from each node so that not much energy is wasted during data communication.

\section{B. Solar energy harvesting circuitry}

Earlier designs of nodes with solar energy harvesting capability used to have rechargeable batteries [18], [19], [20]. With the advancement in the supercapacitors, modern sensor node design incorporates them as their primary energy reservoir of energy and a battery as a secondary energy storage in order to prevent energy outage [21], [22]. Further, in the sensor node design mentioned in [23], [24], [25], [26], only a supercapacitor supports the operation of the node. In all these mentioned works, a solar panel is connected to the energy reservoir (battery or supercapacitor) through an external diode $D_{1}$ as shown in Fig. 1. $D_{1}$ prevents the energy reservoir from discharging through the solar panel and thereby protects solar panel. In order to have a low complexity node, majority of the works on solar harvesting wireless sensor nodes, including commercially available Libelium Waspmote [27], do not use maximum power point tracking based charge controller.

\section{Supercapacitor and solar panel sizing}

In [28], a closed-form expression was developed to obtain the battery size for a solar-energy harvesting WSN node such that the node attains an energy-neutral operation in longterm. However, the parameters required in the closed-form expression have to be found experimentally. Although the work in [15] provides insights on the indoor light intensity and mention that it can be used to find the supercapacitor size, its calculation is not straight forward. In [29], supercapacitor size was computed considering that the energy spent in activities other than communication e.g., in sensing and data logging, is zero. This consideration cannot be used in energy harvesting applications where consumption due to sensing is significantly higher compared to that of radio. The work in [30] reported computation of number of batteries and solar panels that are required to maintain the operation of a solar-powered cellular base stations using average solar intensity. However, these findings cannot be used directly in WSNs due to their significantly different form-factor and power consumption requirements as compared to that of a cellular network. Also for solar harvesting WSN applications, instead of calculating the required number of solar panels, focus should be on the number of solar cells required which make up a solar panel.

\section{System Model AND Problem Definition}

In this section we first outline the WSN architecture considered along with the energy consumption model adopted at the sensor node. Next we discuss the characteristics of solar power communication, followed by the objective of this work.

\section{A. Network Architecture and Energy Consumption Model}

We consider a pollution monitoring application, where static nodes are deployed to sense and store the air quality data of a field, while a mobile entity visits each node sequentially and collects the sensed data. Apart from the basic building blocks, i.e., ultra-low power micro-controller, sensing unit, communication unit, and memory, a field node is considered equipped with a passive wake-up receiver [31], [32], solar panel, and a supercapacitor. The mobile entity contains a wake-up transmitter and a wireless gateway for data collection [33].

In order to increase its lifetime, a sensor node operates in a duty-cycled fashion, i.e., it alternates between sleep state with a very low consumption and active state during which it senses and logs data. The node's sensing duty cycle $\left(D_{c}\right)$ with $N_{s n}$ on-board sensors and sensing rate of $r_{s}$ samples per day is given by $D_{c}=\frac{r_{s}\left(t_{r}^{N_{s n}}+N_{s n} t_{w}\right)}{t_{d}}$, where $t_{r}^{N_{s n}}$ is the sum of response times of $N_{s n}$ sensors, $t_{w}$ is the time for logging one sensor sample and $t_{d}$ is the duration of a day in seconds.

After arriving at the node, the mobile entity sends a wakeup signal. Upon wake-up, the node switches on its radio into receive mode. On successful reception of a hello message from the mobile entity, the node sends an acknowledgement along with the status of stored data. This two-way handshake completes the data transfer by the node. The average power consumption $\left(P_{\text {cons }}^{a v g}\right)$ of a sensor node is given by (1), where $f_{\text {ent }}$ is the frequency of arrival of mobile entity at a node, 


$$
P_{\text {cons }}^{a v g}=\frac{t_{d}}{t_{d}+f_{\text {ent }} t_{o}}\left(\frac{D_{c}\left(t_{r}^{N_{s n}} P_{s e}^{N_{s n}}+N_{s n} t_{w} P_{w}\right)}{t_{r}^{N_{s n}}+N_{s n} t_{w}}+\left(1-D_{c}\right) P_{s l}\right)+\frac{f_{\text {ent }} t_{o} P_{o}}{f_{\text {ent }} t_{o}+t_{d}}
$$

$P_{s e}^{N_{s n}}$ is the average power consumption of $N_{s n}$ sensors during sensing. $P_{w}$ and $P_{s l}$ are the average power consumptions during data logging and sleep state, respectively. $P_{o}$ and $t_{o}$ are respectively the average power and time for communication (both handshaking and sensed data transfer).

\section{B. Solar Powered Communication}

Availability of abundant solar energy during sunlight hours and a solar energy harvester (solar panel) offers the potential for perpetual operation of energy-constrained wireless sensor nodes. From (1) it can be inferred that the node's power consumption increases with the number on-board sensors $N_{s n}$ and the sensing rate $r_{s}$, which ultimately leads to an increase in consumption due to storing and transferring the sensed data. In order to continuously support the operation of node, drained energy of the node's supercapacitor needs to be replenished using the energy harvested by the solar panel.

Energy is harvested via a solar panel, which acts as a voltage limited current source [12]. Generated solar current of a panel at any time is a function of available solar intensity $(G)$. The maximum voltage to which the solar panel can charge an energy storage device is strongly impacted by number of solar cells $\left(N_{S}\right)$ connected in series. Due to loss of the generated energy by the series and the shunt resistances (respectively denoted by $R_{S}$ and $\left.R_{P}\right)$, the output current $\left(I_{M}\right)$ and voltage $\left(V_{M}\right)$ of the solar panel are lesser than their original values at the source.

To characterize the efficacy of solar harvesting system, we first investigate the solar charging characteristics and propose a statistical distribution model for the stochastic solar intensity profile to derive the solar charging rate $(\Gamma)$ of a supercapacitor in a field node. Subsequently, we develop a mathematical formulation for computing the energy outage probability $p_{\text {out }}$ as a function of $\Gamma$ and $P_{\text {cons }}^{a v g}$. Finally, we derive the field node's sizing parameters, i.e., supercapacitor value $C$ and the number of series connected solar cells $N_{S}$.

\section{Solar Charging Rate Characterization}

As shown in Fig. 1, a supercapacitor $C$ can be directly connected across the solar panel in order to store the available solar energy [34]. As alluded in Section II, diode $D_{1}$ protects the solar panel from supercapacitor. We consider $D_{1}$ to be ideal, i.e., the cut-off voltage $V_{x}^{e x t}$ for $D_{1}$ is zero. This is a reasonable assumption as $V_{x}^{\text {ext }}=0.15 \mathrm{~V}$ for a Schottky diode which is typically negligible in comparison with the output voltage $V_{M}$ of a solar panel. Refering to Fig. 1, when this $D_{1}$ is forward biased, the voltage $V_{C}$ across supercapacitor is equal to $V_{M}$, which is a function of solar intensity $G$ and charging time $t$. Solar charging rate $\Gamma$ can be defined as the rate of change of voltage $V_{C}$ across the supercapacitor, i.e., $\Gamma=\frac{d V_{C}}{d t}$. We next discuss the characteristics of a solar panel before deriving $\Gamma$.

\section{A. Circuit Model}

Behavior of the solar panel in standard testing conditions (STC), i.e., with $G=1000 \mathrm{~W} / \mathrm{m}^{2}$, solar cell temperature $T=$ $298 \mathrm{~K}$ can be analyzed from its datasheet parameters, such as, open circuit voltage $\left(V_{O C}\right)$, short circuit current $\left(I_{S C}\right)$, voltage at maximum power $\left(V_{m p}\right)$, current at maximum power $\left(I_{m p}\right)$, and number of solar cells in series $N_{S}$. However, the working of a solar panel at any given operating conditions can be analyzed only using the equivalent circuit models for solar cells as in [12] [13]. Solar panel with $N_{S}$ solar cell unit in series acts as a resultant solar cell with series resistance, shunt resistance, and diode ideality factor $N_{S}$ times that of the original solar cell unit [12]. Using Kirchoff's laws for the equivalent circuit of solar panel shown in Fig. 1, it can be inferred that the solar panel output voltage $V_{M}$ and current $I_{M}$ are governed by the following relationships:

$$
I_{M}=I_{i r r}-I_{0}\left(\exp \left[\frac{q V_{R_{P}}}{N_{S} n k T}\right]-1\right)-\frac{V_{R_{P}}}{N_{S} R_{P}}
$$

where $V_{R_{P}}=V_{M}+I_{M} N_{S} R_{S}, k$ and $q$ are respectively the Boltzmann's constant and charge of an electron, $I_{0}$ and $n$ denote the reverse saturation current and ideality factor for diode $D_{1}$. The unknown circuit parameters $n, I_{i r r}, I_{0}, R_{S}, R_{P}$ at any operating conditions are related to the circuit parameters at STC (super-scripted by $S T C$ ) as follows [12]:

$$
\begin{gathered}
I_{i r r}=I_{i r r}^{S T C}\left(\frac{G}{G^{S T C}}\right)\left(1+\alpha_{T}^{\prime}\left(T-T^{S T C}\right)\right), \\
I_{0}=I_{0}^{S T C}\left(\frac{T}{T^{S T C}}\right)^{3} \exp \left[\frac{E_{g}^{S T C}}{k T^{S T C}}-\frac{E_{g}}{k T}\right], \\
n=n^{S T C}, R_{S}=R_{S}^{S T C}, R_{P}=R_{P}^{S T C}\left(\frac{G}{G^{S T C}}\right),
\end{gathered}
$$

where $\alpha_{T}^{\prime}$ in (3a) is the relative temperature coefficient of short circuit current and $E_{g}$ in (3b) is the band gap energy of silicon diode, given by: $E_{g}=1.16-7.02 \times 10^{-4} T^{2}(T-1108)^{-1}$.

For small solar panels used in wireless sensor networks, $\alpha_{T}^{\prime}$ is rarely given in the datasheet. In [12] it has been experimentally found out that $\alpha_{T}^{\prime} \approx 0$, which we consider in our subsequent development. Further, we also assume $n^{S T C}=1.3$ as suggested in [35]. The remaining four circuit parameters at STC $\left(I_{i r r}^{S T C}, I_{0}^{S T C}, R_{S}^{S T C}, R_{P}^{S T C}\right)$ can be extracted by substituting datasheet parameters in three equations obtained from equation (2) at the short circuit condition $\left(V_{M}=0, I_{M}=I_{S C}\right)$, open circuit condition $\left(I_{M}=0, V_{M}=V_{O C}\right)$, and the maximum power point condition $\left(V_{M}=V_{m p}, I_{M}=I_{m p}\right)$. Finally the expression of $R_{P}^{S T C}$ is obtained at maximum power point using the condition $\left(\frac{d P}{d V_{M}}=0, V_{M}=V_{m p}, I_{M}=I_{m p}\right)$ and substituting datasheet parameters in it. Note that $P=V_{M} I_{M}$ is the power 
of solar panel. Further, after re-arranging the terms in (2), $I_{M}$ can be written as a function of $V_{M}$ as:

$$
I_{M}=\frac{A-B}{q N_{S} R_{S} R_{e f f}},
$$

where

$$
A=q R_{S}\left(I_{e f f} N_{S} R_{P}-V_{M}\right),
$$

$$
\begin{gathered}
B=k n T N_{S} R_{e f f} \mathrm{~W}\left[\frac{\exp \left[\frac{q R_{P}\left[I_{e f f} N_{S} R_{S}+V_{M}\right]}{k n N_{S} T R_{e f f}}\right] q I_{0} R_{P} R_{S}}{k n R_{e f f} T}\right], \\
I_{\text {eff }}=I_{0}+I_{i r r}, \quad(4 \mathrm{~b}) \\
R_{\text {eff }}=R_{S}+R_{P}, \quad(4 \mathrm{~d})
\end{gathered}
$$

and $W(\cdot)$ is the Lambert function [36].

For gaining more insights on the solar panel behaviour, we consider a special case of solar panel that is discussed next.

\section{B. Solar Charging Rate for Practical Solar Panel Model}

Since the supercapacitor is in series with the solar panel when $D_{1}$ is forward biased, input charging current of the supercapacitor $\left(I_{C}\right)$ is same as that of the output current $I_{M}$ of the solar panel. Therefore, $I_{C}=I_{M}=C \frac{d V_{C}}{d t}$.

From equations (9) and (4a) we have the solar charging rate $\Gamma_{p}$ for the practical solar panel model as:

$$
\Gamma_{p}=\frac{d V_{C}}{d t}=\left.\frac{A-B}{q N_{S} R_{S} R_{e f f} C}\right|_{V_{M}=V_{C}} .
$$

Useful insights can be obtained when the composite Lambert function present in the expression of $B$ (cf. (5) and (4c)) is replaced with a simpler function. Next, we propose an approximation function for the composite Lambert function. Additionally, we show later how it can be used to analytically derive the output voltage of the solar panel until which the approximate solar panel model is valid.

Proposition 1. The composite Lambert function of $V_{C}$ in $(4 c)$ can be approximated with a Gaussian function as:

$B \approx k n T N_{S} R_{e f f}\left(a_{11} G+a_{12}\right) \exp \left[-\left(\frac{V_{C}-b_{1}^{a v g}}{c_{1}^{a v g}}\right)^{2}\right]$,

where $a_{11}, a_{12}, b_{1}^{a v g}, c_{1}^{\text {avg }}$ are functions of solar intensity $G$.

Proof. Below, we propose a tight Gaussian approximation for the composite Lambert function in $B$ as defined in $(4 \mathrm{c})$.

$$
\frac{B}{k n T N_{S} R_{e f f}} \approx a_{1} \exp \left[-\left(\frac{V_{C}-b_{1}}{c_{1}}\right)^{2}\right],
$$

Here the variable $a_{1}$ is a linear function of solar intensity $G$ and variables $b_{1}$ and $c_{1}$ have negligible minor variations with $G$. Therefore, the Gaussian function shown in right hand side of equation (7) can be replaced by a similar Gaussian function with new values of $a_{1}$ (denoted by $a_{1}^{\text {new }}$ ), and both $b_{1}$, $c_{1}$ replaced with their average values $b_{1}^{a v g}, c_{1}^{a v g}$, respectively. Moreover, it is found that $a_{1}^{\text {new }}$ can be fitted into a linear

\begin{tabular}{|c|c|c|}
\hline Parameter & Symbol & Value \\
\hline \multicolumn{3}{|c|}{ Parameters of solar panel from datasheet } \\
\hline Maximum power & $P_{\max }^{S T C}$ & $3 \mathrm{~W}$ \\
\hline Voltage at $P_{\max }^{S T C}$ & $V_{m p}^{S T C}$ & $6.9 \mathrm{~V}$ \\
\hline Current at $P_{\max }^{S T C}$ & $I_{m p}^{S T C}$ & $0.44 \mathrm{~A}$ \\
\hline Short circuit current & $I_{S C}^{S T C}$ & $0.47 \mathrm{~A}$ \\
\hline Open circuit voltage & $V_{O C}^{S T C}$ & $8.49 \mathrm{~V}$ \\
\hline Number of cells & $N_{S}$ & 14 \\
\hline \multicolumn{3}{|c|}{ Derived parameters of solar cell unit } \\
\hline Series resistance & $R_{S}^{S T C}$ & $0.04891 \Omega$ \\
\hline Parallel resistance & $R_{P}^{S T C}$ & $2207.82 \Omega$ \\
\hline Solar current & $I_{i r r}^{S T C}$ & $0.47001 \mathrm{~A}$ \\
\hline Reverse saturation current & $I_{0}^{S T C}$ & $6.0234 \mathrm{nA}$ \\
\hline
\end{tabular}
function of $G$, i.e., $a_{1}^{\text {new }} \approx a_{11} G+a_{12}$, where $a_{11}$ and $a_{22}$ are constants. So, (7) can be rewritten as:

$a_{1} \exp \left[-\left(\frac{V_{C}-b_{1}}{c_{1}}\right)^{2}\right] \approx\left(a_{11} G+a_{12}\right) \exp \left[-\left(\frac{V_{C}-b_{1}^{a v g}}{c_{1}^{a v g}}\right)^{2}\right]$.
TABLE I: System parameters for $3 \mathrm{~W}$ panel at STC.

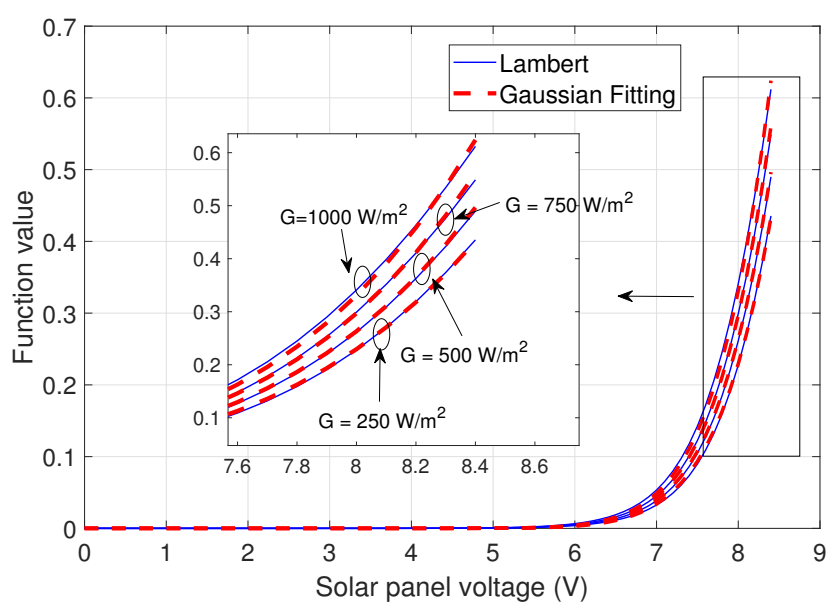

Fig. 2: Gaussian fitting function for Lambert function of $V_{C}$.

Validity of this proposition is demonstrated for a $3 \mathrm{~W}$ solar panel whose parameters are listed in Table I. The temperature of solar cell is considered to be $T=298 \mathrm{~K}$. As shown in equation (3a), $I_{i r r}$ and $R_{P}$ vary with solar intensity $G$. Simulation results suggest that the parameters $b_{1}$ and $c_{1}$ respectively can be replaced with their averages $b_{1}^{a v g}=11.62$, $c_{1}^{a v g}=2.076$, whereas $a_{1}^{n e w}=0.002828 G+4.091$ is obtained by using a linear fit for $a_{1}$ as a function of solar intensity $G$. Fig. 2 shows the composite Lambert function and its corresponding Gaussian function for different values of $G$ obtained using $b_{1}^{a v g}, c_{1}^{a v g}$ and the the linear fit for $a_{1}^{n e w}$. Inset figure clearly shows a good match between the composite Lambert function in $B$ and the Gaussian fitting function.

Next, to gain further analytical insights on charging rate in practical solar panel model we propose a tight approximation.

\section{Approximate Solar Panel Model}

To get a closed-form expression for solar charging rate we propose an approximation for practical solar panel model following the approximate diode model [37] for diode $D_{2}$. 
As per the approximate diode model, the reverse saturation current $I_{0}$ for diode $D_{2}=0$ below its cut-off voltage $\left(V_{x}^{i n t}\right)$. As a number of solar cells are connected in series inside the solar panel, $D_{2}$ typically has a cut-off voltage $V_{x}^{\text {int }}$ which is much higher than the cut-off voltage $V_{x}^{\text {ext }}$ of $D_{1}$. Next we explain how the expression for solar charging rate gets simplified when $D_{2}$ is either reverse biased or forward biased with $I_{0}=0$ assumption.

By putting $I_{0}=0$ in (4a), the output voltage $V_{M}$ and current $I_{M}$ in approximate solar panel model are related as:

$$
I_{M}=\frac{I_{i r r} N_{S} R_{P}-V_{M}}{N_{S} R_{e f f}} .
$$

Fig. 3 shows the $V_{M}-I_{M}$ characteristics of a $3 \mathrm{~W}$ solar panel; the panel parameters are listed in Table I. At different intensities the performance of practical and approximate solar panel models are shown using solid and dashed lines, respectively. Square boxes in Fig. 3 indicate the cut-off voltage $V_{x}^{\text {int }}$ of $D_{2}$ of solar panel which is determined considering that the output current difference between the two solar panel models is less than $10^{-3}$. Note that, $V_{x}^{\text {int }}>5 \mathrm{~V}$, which is much higher than the cut-off of $D_{1} V_{x}^{e x t}=0.15 \mathrm{~V}$ for zener diode.

When forward bias voltage of the diode $D_{2}$ is below $V_{x}^{i n t}$, $D_{2}$ acts as an open circuit, during which most of the generated solar current $I_{i r r}$ flows to the supercapacitor due to a large value of shunt resistance $R_{P}$. However, when the forward bias of the $D_{2}$ is above $V_{x}^{i n t}, D_{2}$ acts as a short circuit and all the generated solar current flows through it. Therefore, when $D_{1}$ is forward biased, the supercapacitor charges only during the reverse bias of $D_{2}$ and the maximum voltage $\left(V_{C}^{\max }\right)$ to which the supercapacitor charges is equal to $V_{x}^{i n t}$.

After rearranging the terms of (9) and integrating, we get:

$$
t_{c}^{a}=C N_{S} R_{e f f} \ln \left(\frac{N_{S} R_{P} I_{i r r}-V_{r e s}}{N_{S} R_{P} I_{i r r}-V_{C}}\right),
$$

where $t_{c}^{a}$ is the time taken for the supercapacitor to charge from its residual voltage $V_{\text {res }}$ to final voltage $V_{C}$. Note that (10) is valid only when $V_{C}>V_{\text {res }}$, i.e., when $D_{1}$ is forward biased. After re-arranging the terms in (10), $V_{C}$ can be written as a function of time $t$ or $t_{c}^{a}$. So, $V_{C}=$

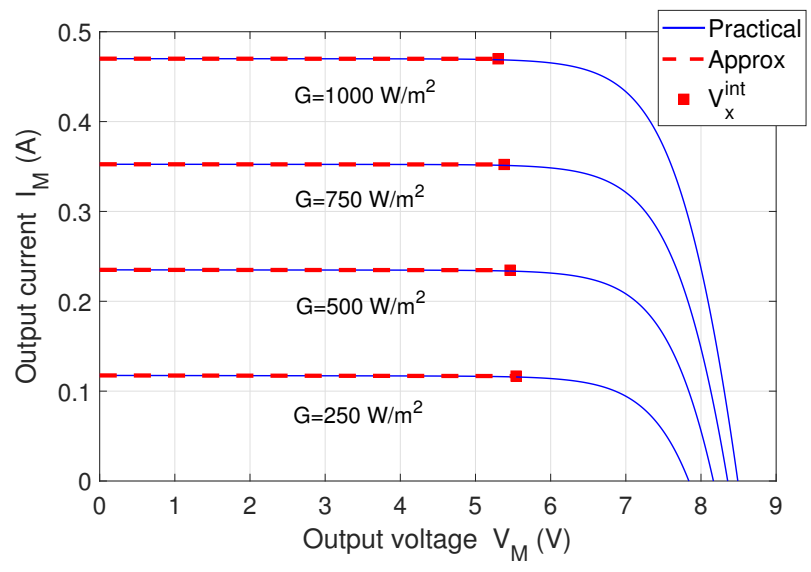

Fig. 3: Output characteristics of solar panel.
$\left(V_{\text {res }}-N_{S} R_{P} I_{i r r}\right) \exp \left[\frac{-t}{C N_{S} R_{\text {eff }}}\right]+N_{S} R_{P} I_{\text {irr }}$ when the $D_{1}$ forward biased and $V_{C}=V_{\text {res }}$ when the $D_{1}$ reverse biased. Based on this, the solar charging rate $\Gamma_{a}$ for approximate solar panel model is given as:

$$
\Gamma_{a}=\frac{d V_{C}}{d t}=\frac{N_{S} R_{P} I_{i r r}-V_{r e s}}{C N_{S} R_{e f f}} \exp \left[\frac{-t}{C N_{S} R_{e f f}}\right] .
$$

The approximate model of solar panel is obtained by using the approximate model for the diode $D_{2}$ whose reverse saturation current is 0 below its cut-off voltage $V_{x}^{\text {int }}$. From Fig. 1, it can be seen that the voltage across $D_{2}$ is dependent on voltage $V_{C}$ of the supercapacitor $C$. Since we assumed $D_{1}$ as an ideal diode, when the $V_{C}<V_{x}^{i n t}, D_{2}$ is reverse-biased. Therefore, no solar current flows through $D_{2}$ and during this time the supercapacitor $C$ charges. Moreover, it ceases to charge once its voltage $V_{C}$ close to $V_{x}^{\text {int }}$, during which all the solar current flows through the diode $D_{2}$. Therefore, the closed form equation of supercapacitor voltage evolution is valid only when the output solar panel voltage $V_{M}$ is below $D_{2}$ 's cut-off voltage $V_{x}^{\text {int }}$. $V_{x}^{\text {int }}$ can be found by making the current across the $D_{2}$ to be negligible. In other words, $V_{x}^{\text {int }}$ is the bias voltage value until when $B$ (defined in (4c)) is negligible with respect to $A$ (defined in (4b)). This can be mathematically written as:

$$
B \ll A, \text { or }, B<\min \{A\} .
$$

We get $\min \{A\}$ when $V_{C}=V_{x}^{\text {int }}$. By considering that $B$ is always below the lowest value of $A$ by an acceptable value $x$, (12) can be re-written as:

$$
B=\min \{A\}-x .
$$

By equating (13) and (6) and re-arranging the terms, we get the maximum voltage $V_{C}^{\max }$ up to which a supercapacitor is charged using the approximate solar panel model:

$$
V_{C}^{\max }=b_{1}^{a v g}+c_{1}^{a v g} \sqrt{\ln \left[\frac{k n T N_{S} R_{e f f}\left(a_{11} G+a_{12}\right)}{\min \{A\}-x}\right]} .
$$

Note that $V_{C}^{\max }$ is equal to $V_{x}^{\text {int }}$ since $V_{C}^{\max }$ is also same as the maximum voltage till which approximate solar panel model is valid. This development will be used later in Section VI for the harvested energy characterization.

\section{Proposed Solar Intensity Distribution}

In this section we model the empirical solar intensity readings obtained from the publicly available data-sets provided by National Renewable Energy Laboratory (NREL) [38].

\section{A. Empirical Dataset for Solar Intensity Profile}

A solar intensity distribution model can be developed based on the intensity data collected at different times of day [30]. To this end, from the solar intensity dataset of a particular location obtained from the NREL dataset [38], we develop a single distribution model for characterizing the intensity profile. For example, we consider all available hourly intensity $G$ values for New Delhi, over 15 years $(2000-2014)$ to find a single solar panel size that is suitable for all solar intensity conditions. We next present how these samples can be well fitted into a polynomial-fit based mixture distribution. 


\section{B. Polynomial-fit Based Mixture Distribution}

We observe that the solar intensity $G=0$ during night. As this corresponds to nearly half the 24-hour duration of a day, the probability of solar intensity being 0 is maximum $(\approx$ 0.5 ) out of all other intensity values. Therefore, the probability density function (PDF) of solar intensity $G$ is captured by the summation of weighted dirac-delta function $\delta(\cdot)$ defined for the solar intensity $G$ value 0 and the weighted polynomial function $f_{p}$ defined over rest of the positive intensity values. $\operatorname{PDF} f_{G}^{p o l}=\operatorname{Pr}[G=g]$ of solar intensity $G$ is given by:

$$
f_{G}^{p o l}(g)=w_{d} \delta(g)+w_{p} f^{p o l}(g) \text {, with } w_{d}+w_{p}=1,
$$

Here $w_{d}, w_{p}$ are the positive weights associated respectively with dirac-delta and polynomial functions, and they represent the relative frequencies of various solar intensity values. For example, the available data from New Delhi shows that the probability of occurrence of solar intensity value $g=0$ is $w_{d}=0.5076$. Thus, $w_{p}=0.4924$. Further,

$$
\begin{gathered}
\delta(g)= \begin{cases}1, & g=0 \\
0, & \text { otherwise, }\end{cases} \\
f^{p o l}(g)=\sum_{i=0}^{m} q_{i} g^{m-i}, \quad 0<g \leq \max \{g\} .
\end{gathered}
$$

where $m$ denotes the order of polynomial function and $q_{i}$ is the $i^{t h}$ coefficient of the polynomial.

\section{Validation of Proposed Distribution Model}

The order of the polynomial and its coefficients depend on the required goodness-of-fit of the distribution. We have performed fitting using least squares algorithm so that the area under the curve over $0<G<\max \{G\}$ is equal to 1 . From the available New Delhi data set with $\max \{G\}=988$, Fig. 4 shows the polynomial fit of solar intensity with $m=20$. Goodness-of-fit parameter values of 0.000088 and 0.999969 respectively in terms of Root Mean Square Error and R-square validate that there is negligible error between the actual and the polynomial-fit based mixture distribution.

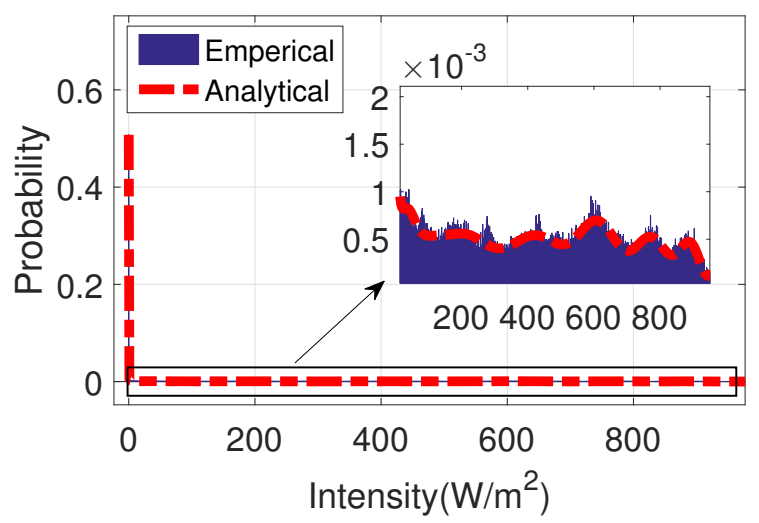

Fig. 4: Validation of polynomial-fit based mixture distribution of $G$.
The cumulative distribution function (CDF) $F_{G}^{\text {pol }}$ of solar intensity at New Delhi region using a polynomial-fit based mixture distribution is given by:

$$
\begin{aligned}
& F_{G}^{p o l}(\varphi)=\operatorname{Pr}(G \leq \varphi)=\int_{0}^{\varphi} f_{G}^{p o l}(g) d g \\
& = \begin{cases}0 & \varphi<0 \\
0.5076 & 0 \leq \varphi<0^{+} \\
0.5076+0.4924 \sum_{i=0}^{20} \frac{q_{i} \varphi^{21-i}}{21-i} & 0^{+} \leq \varphi \leq 988 \\
1 & \varphi \geq 988 .\end{cases} \\
& \text { Vi. Stochastic Harvested Energy }
\end{aligned}
$$

\section{Stochastic HaRVESTEd ENERGy}

For harvested energy characterization, we first derive the solar charging rate distribution with both approximate and practical models, where we use the results of circuit analysis in Section IV and solar energy distribution model proposed in Section V. Then, we derive the energy outage probability and estimate the practical supercapacitor and panel sizes to enable sustainable solar harvesting network operation.

\section{A. Solar Charging Rate Distribution}

1) Solar Charging Rate Distribution $F_{\Gamma_{a}}$ using Approximate Solar Panel Model: Using (11), the CDF $F_{\Gamma_{a}}\left(\gamma_{a}\right)$ of charging rate $\Gamma_{a}$ in case of approximate solar panel model is given by:

$$
\begin{aligned}
F_{\Gamma_{a}}\left(\gamma_{a}\right) & =\operatorname{Pr}\left[\Gamma_{a} \leq \gamma_{a}\right] \\
& =\operatorname{Pr}\left[I_{i r r} \leq \frac{V_{\text {res }}}{N_{S} R_{P}}+\frac{\gamma_{a} C R_{e f f}}{R_{P}} \exp \left[\frac{t}{C N_{S} R_{e f f}}\right]\right]
\end{aligned}
$$

which on using (3a) reduces to:

$$
F_{\Gamma_{a}}\left(\gamma_{a}\right)=\operatorname{Pr}[G \leq \zeta]=F_{G}(\zeta)
$$

Here $F_{G}$ is the CDF of solar intensity $G$, and $\zeta$ is defined as:

$$
\zeta \triangleq \frac{G^{S T C}\left(V_{\text {res }}+\gamma_{a} N_{S} C R_{e f f} \exp \left[\frac{t}{C N_{S} R_{e f f}}\right]\right)}{N_{S} R_{P} I_{i r r}^{S T C}\left(1+\alpha_{T}^{\prime}\left(T-T^{S T C}\right)\right)}
$$

2) Solar Charging Rate Distribution $F_{\Gamma_{p}}$ using Practical Solar Panel Model: $F_{\Gamma_{p}}\left(\gamma_{p}\right)$ is derived similarly as the derivation of $F_{\Gamma_{a}}\left(\gamma_{a}\right)$. To obtain a closed-form expression for $F_{\Gamma_{p}}\left(\gamma_{p}\right)$, we use the tight approximation (7) for the composite Lambert function in (5). The $\mathrm{CDF} F_{\Gamma_{p}}\left(\gamma_{p}\right)$ of solar charging rate $\Gamma_{p}$ is given by:

$$
F_{\Gamma_{p}}\left(\gamma_{p}\right)=\operatorname{Pr}\left[\Gamma_{p} \leq \gamma_{p}\right]=\operatorname{Pr}[G \leq \nu]=F_{G}(\nu)
$$

where $\nu$ is given by (18b) in which $V_{C}$ depends on $\Gamma_{p}$ (cf. (5)). 


$$
\nu=\frac{q R_{S}\left[V_{C}-I_{0} N_{S} R_{P}\right]+G^{S T C} N_{S} R_{e f f}\left[\gamma_{p} q R_{S} C+k n T a_{12} \exp \left(-\left[\frac{V_{C}-b_{1}^{a v g}}{c_{1}^{a v g}}\right]^{2}\right)\right]}{q N_{S} R_{P} R_{S} I_{i r r}^{S T C}\left[1+\alpha_{T}^{\prime}\left(T-T^{S T C}\right)\right]-k n T N_{S} R_{e f f} a_{11} G^{S T C} \exp \left(-\left[\frac{V_{C}-b_{1}^{a v g}}{c_{1}^{a v g}}\right]^{2}\right)}
$$

\section{B. Energy Outage Probability ( $\left.p_{\text {out }}\right)$}

If $V_{C}(t)$ is the voltage across the supercapacitor at time $t$, the voltage at time $t+\Delta t$ is given by:

$$
\begin{aligned}
V_{C}(t+\Delta t)= & V_{C}(t)+\Gamma \Delta t \\
& -\left(V_{C}(t)-\sqrt{V_{C}^{2}(t)-\frac{2 P_{\text {cons }}^{a v g} \Delta t}{C}}\right) \\
= & \Gamma \Delta t+\sqrt{V_{C}^{2}(t)-\frac{2 P_{\text {cons }}^{\text {avg }} \Delta t}{C}} .
\end{aligned}
$$

where $P_{\text {cons }}^{a v g}$ is the average power consumption of the wireless sensor node and $\Gamma$ is the solar charging rate. Energy outage occurs when $V_{C}(t+\Delta t)$ is lower than the minimum threshold voltage $V_{l}$ of the supercapacitor, or in other words, when $\Gamma<$ $\Psi$, where:

$$
\Psi \triangleq \frac{V_{l}-\sqrt{V_{r e s}^{2}-\frac{2 P_{c o n s}^{a v g} \Delta t}{C}}}{\Delta t},
$$

where, $V_{\text {res }}$ is the residual voltage of supercapacitor. Using (20a), the energy outage probability $p_{\text {out }}$ can be written as:

$$
p_{\text {out }}=\operatorname{Pr}[\Gamma<\Psi]=F_{\Gamma}(\Psi) .
$$

Therefore, $p_{\text {out }}$ in the two cases of the model are given by:

$$
p_{\text {out }}= \begin{cases}F_{\Gamma_{a}}(\Psi)=\left.F_{G}(\zeta)\right|_{\gamma_{a}=\Psi}, & \text { Approximate } \\ F_{\Gamma_{p}}(\Psi)=\left.F_{G}(\nu)\right|_{\gamma_{p}=\Psi}, & \text { Practical. }\end{cases}
$$

\section{Solar Panel Size Estimation}

The load connected in parallel to the supercapacitor of Fig. 1 needs a minimum voltage $V_{\text {load }}^{\text {min }}$ to operate, and it works till the voltage across it is within a maximum $V_{\text {load }}^{\max }$. For example, Waspmote [27] operates between $V_{\text {load }}^{\mathrm{min}}=3.3 \mathrm{~V}$ and $V_{\text {load }}^{\max }=4.2 \mathrm{~V}$. Similarly, there is a lower voltage limit $V_{C}^{\text {min }}$ below which capacitor should not be discharged, while it should not be charged above a maximum voltage level $V_{C}^{\max }$. For example, a Taiyo Yuden LIC1235RS3R8406 supercapacitor [39] has $V_{C}^{\min }=2.2 \mathrm{~V}$ and $V_{C}^{\max }=3.8 \mathrm{~V}$. We define the voltages $V_{\max }$ and $V_{\min }$ as follows:

$$
\begin{aligned}
& V_{\text {max }} \triangleq \min \left\{V_{C}^{\max }, V_{\text {load }}^{\max }\right\} \\
& V_{\text {min }} \triangleq \max \left\{V_{C}^{\min }, V_{\text {load }}^{\min }\right\} .
\end{aligned}
$$

For an energy harvesting wireless sensor node deployed at a location whose maximum solar intensity is $G_{\max }$, the number of series connected solar cells $N_{S}^{p}$ needed in case of a practical solar panel model can be found by substituting $V_{M}=V_{\max }+V_{x}^{e x t}, I_{M}=0, T=T_{\text {ref }}, G=G_{\max }$ in (2), and solving for $N_{S}^{p}$. The conditions used to find $N_{S}^{p}$ for practical solar panel ensures that the supercapacitor can be charged up to a maximum voltage of $V_{\max }$.
In order to ensure the validity of approximate solar panel model in an average sense and also charge the supercapacitor to a maximum voltage $V_{\max }$, solar panel size $N_{S}^{a}$ should be such that during sunlight hours, on an average, output voltage $V_{M}$ of the solar panel has a linear current characteristic for all the voltages in the range $\left[V_{\min }, V_{\max }\right]$. By substituting $V_{M}=V_{\max }, I_{M}=I_{i r r}-\Delta I, T=T^{S T C}, G=G_{\max }$ in (2), the unknown quantity $N_{S}^{a}$ can be calculated. Note that $\Delta I$ is a small value compared to $I_{i r r}$.

\section{Supercapacitor Size Estimation}

For a fixed solar panel size, we first calculate the supercapacitor size $C=C_{1}$ such that the excess energy can be stored. Excess energy is the difference between the total energy that can be harvested and the energy consumed by the load during the time when sunlight is available. Additionally, we select the supercapacitor's size such that at time $t=t_{\text {start }}$ (i.e., at the start of day light time) it begins to charge from a voltage $V_{C}\left(t_{\text {start }}\right)=V_{\min }$, and by the end of sunlight time $t=t_{\text {end }}$ the supercapacitor builds up its voltage to $V_{C}\left(t_{\text {end }}\right)=V_{\max }$.

By taking expectation on both sides of (19) we get:

$$
\mathbb{E}\left[V_{C}(t+\Delta t)\right]=\mathbb{E}[\Gamma] \Delta t+\mathbb{E}\left[\sqrt{V_{C}(t)-\frac{2 P_{\text {cons }}^{\text {avg }} \Delta t}{C_{1}}}\right],
$$

where, $\forall t \in\left[t_{\text {start }}, t_{\text {end }}-\Delta t\right]$ and the average solar charging rate $\mathbb{E}[\Gamma]$ is a function of capacitor size, intensity profile, and solar panel parameters.

The details for finding $C_{1}$ using the update equation (22) for an approximate solar panel model are provided in Algorithm 1.

$\overline{\text { Algorithm } 1 \text { Estimating } C_{1} \text { for approximate solar panel }}$ model.

Input: Solar intensity dataset $\mathbf{D}$, solar panel parameters, $V_{\max }, V_{\min }, P_{\text {cons }}^{\text {avg }}$

Output: Supercapacitor size $C_{1}$

$$
\begin{aligned}
& \text { 1: } t_{\mathrm{end}}=\Delta t\left\lceil\frac{86400}{\Delta t} \times \frac{|\mathbf{D}>0|}{|\mathbf{D}|}\right\rceil \\
& \text { 2: } Z_{1}=\frac{R_{P} I_{i r r}^{S T C}}{C_{1} R_{e f f} G^{S T C}} \exp \left(\frac{-\Delta t}{C_{1} N_{S} R_{e f f}}\right) \\
& \text { 3: } Z_{2}=\frac{1}{C_{1} N_{S} R_{e f f}} \exp \left(\frac{-\Delta t}{C_{1} N_{S} R_{e f f}}\right) \\
& \text { 4: Set } t \leftarrow 0, V_{C}(t) \leftarrow V_{\min } \\
& \text { 5: for } i \in\left\{1, \ldots, \frac{t_{\text {end }}}{\Delta t}\right\} \text { do } \\
& \text { 6: } \quad \mathbb{E}\left[\Gamma_{a}\right] \leftarrow Z_{1} \mathbb{E}[G>0]-Z_{2} V_{C}(t+(i-1) \Delta t) \\
& \text { 7: } \mathbb{E}\left[V_{C}(t+i \Delta t)\right] \leftarrow \mathbb{E}[\Gamma] \Delta t+ \\
& \sqrt{V_{C}(t+(i-1) \Delta t)-\frac{2 P_{\text {cons }}^{\text {avg }} \Delta t}{C_{1}}} \\
& \text { 8: } \quad V_{C}(t+i \Delta t) \leftarrow \mathbb{E}\left[V_{C}(t+i \Delta t)\right] \\
& \text { 9: Solve } V_{C}\left(t_{\text {end }}\right)=V_{\max } \text { to find } C_{1}
\end{aligned}
$$


Although (22) is generic in nature, for practical solar panel model, solar charging rate $\Gamma_{p}$ can only be expressed in terms of $V_{C}(t+\Delta t)$ instead of $V_{C}(t)$ and $\Delta t$. Hence, to find $C_{1}$ suitable for a practical solar panel, we re-write (19) by replacing $t$ with $t-\Delta t$ and take an expectation on both sides to get:

$$
\mathbb{E}\left[V_{C}(t-\Delta t)\right]=\mathbb{E}\left[\sqrt{\left(V_{C}(t)-\Gamma_{p} \Delta t\right)^{2}+\frac{2 P_{\text {cons }}^{\text {avg }} \Delta t}{C_{1}}}\right]
$$

$\forall t \in\left[t_{\text {start }}+\Delta t, t_{\text {end }}\right]$. As solar charging rate $\Gamma_{p}$ for a practical solar panel is a complex function of solar intensity $G$ (cf. (5), (4b), and (6)), the right hand side of (23) is hard to compute after transformation of random variable $G$. Next we discuss some approximations to find the bounds for $C_{1}$.

The upper bound $C_{1}^{u}$ is obtained when the available solar energy is stored such that there is no consumption by the sensor node, i.e., $P_{\text {cons }}^{a v g}=0$. Thus, for a practical solar panel model with charging rate $\Gamma_{p},(23)$ is re-written as:

$\mathbb{E}\left[V_{C}(t-\Delta t)\right]=\mathbb{E}\left[V_{C}(t)\right]-\mathbb{E}\left[\Gamma_{p}\right] \Delta t \quad \forall t \in\left[t_{\text {start }}+\Delta t, t_{\text {end }}\right]$

By considering a non-zero average energy consumption, i.e., $P_{\text {cons }}^{a v g} \neq 0$, the amount of solar energy to be harvested is lower compared to the case when $P_{\text {cons }}^{a v g}=0$. As a result, a smaller supercapacitor $C_{1}^{l}$ (lower bound) would be required.

As the terms $\left(V_{C}(t)-\Gamma_{p} \Delta t\right)$ and $\sqrt{\frac{2 P_{\text {cons }}^{a v g} \Delta t}{C_{1}}}$ are positive, the following relationship holds:

$$
\sqrt{\left(V_{C}(t)-\Gamma_{p} \Delta t\right)^{2}+\left(\sqrt{\frac{2 P_{C_{c o n s}}^{a v g} \Delta t}{C_{1}}}\right)^{2}}<\left(V_{C}(t)-\Gamma_{p} \Delta t\right)+\sqrt{\frac{2 P_{c o n s}^{a v g_{s} \Delta t}}{C_{1}}} .
$$

By taking expectation on both sides of (25a) and using (23),

$\mathbb{E}\left[V_{C}(t-\Delta t)\right]<\mathbb{E}\left[V_{C}(t)\right]-\mathbb{E}\left[\Gamma_{p}\right] \Delta t+\sqrt{\frac{2 P_{\text {cons }}^{a v g} \Delta t}{C_{1}}}$

$\forall t \in\left[t_{\text {start }}+\Delta t, t_{\text {end }}\right]$. The detailed pseudo code for finding the bounds on supercapacitor size $C_{1}$ using the update equations (24) and (25b) is provided in Algorithm 2.

Given the solar intensity distribution at a particular location, parameters of the solar panel and the average power consumption $P_{\text {cons }}^{a v g}$ of the wireless sensor node connected to supercapacitor, Algorithm 2 estimates the upper and lower bounds of the required supercapacitor. For this, we start with the assumption that at the end of the day, supercapacitor will be at its maximum voltage $V_{\max }$. Update equations (24) and (25b) are used to iterate through time in steps of $\Delta t$ such that voltage at beginning of the day is $V_{\min }$. In Algorithm 2, it is shown that first the lower bound and next the higher bound are calculated sequentially. However, they can be computed parallely, since both are independent of each other. It is presented this way so that we do not mention the same algorithm twice. Since the algorithm takes $\frac{t_{\text {end }}}{\Delta t}$ iterations to compute, the time complexity of the algorithm is $O\left(\frac{t_{\text {end }}}{\Delta t}\right)$, where $\Delta t$ is an independent quantity, the lower the value, more accurate result would be obtained at the cost of more time. $t_{\text {end }}$ (in seconds) is day-light hours of a particular location.

Next, we find the supercapacitor size $C_{2}$ by ensuring that it is large enough to support the average load consumption over $\overline{\text { Algorithm } 2 \text { Estimating bounds on super-capacitor size } C_{1}}$ for practical solar panel.

Input: Solar intensity dataset $\mathbf{D}$, panel parameters, $P_{\text {cons }}^{\text {avg }}$

Output: Supercapacitor size $C_{1} \in\left[C_{1}^{l}, C_{1}^{u}\right]$

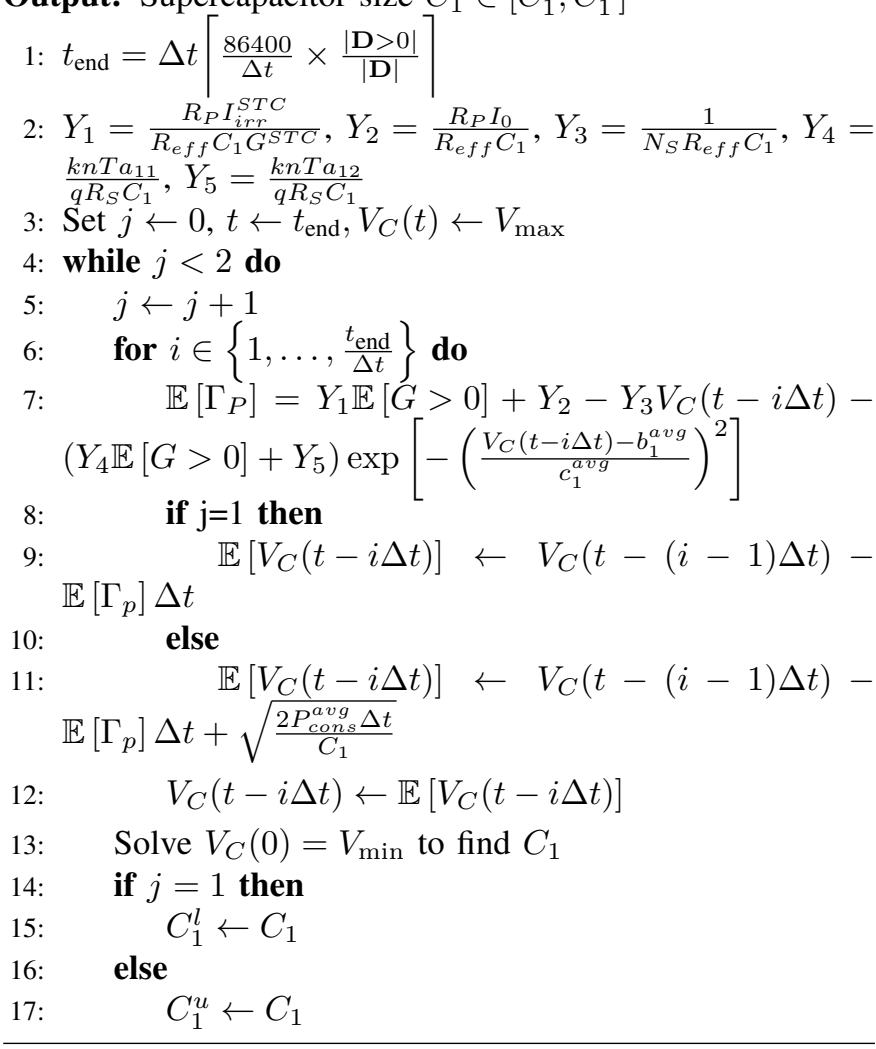

the non-sunlight hours using the energy that is stored at the beginning of non-sunlight hours, i.e., at $t=t_{\text {end }}$. We assume that $V_{C}(t)=V_{\min }$ at $=t_{\text {start }}$, and $V_{C}(t)=V_{\max }$ at $t=t_{\text {end }}$. Mathematically, $C_{2}$ is given by:

$$
C_{2}=\frac{2 P_{c o n s}^{a v g}}{V_{\max }^{2}-V_{\min }^{2}} \times\left(t_{\mathrm{end}}-t_{\mathrm{start}}\right) .
$$

\section{ViI. Performance Evaluation and Discussion}

The mathematical analysis presented till now is validated using a bottom-up approach. First, the solar panel characteristics presented in Fig. 3 are validated by comparing the

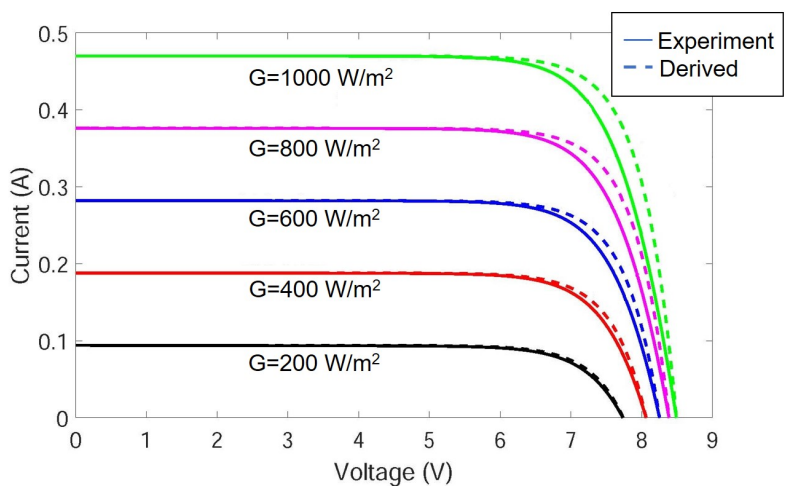

Fig. 5: Experimental validation of solar panel characteristics. 
I-V characteristics of solar panel using experiments. Next, the results for consumptions of various components of the Waspmote are presented, which were found to be in good agrrement to the results presented in its datasheet [27]. Later, the mathematical analysis presented on the solar charging rate $\mathrm{CDF}$ and the energy outage probability of the solar-energy harvesting Waspmote are validated using simulations with the help of solar intensity readings obtained for NREL [12] database. Towards the end of the section, results on sizing of both solar panel and supercapacitor have been presented.

I-V characteristic of solar panel under test is found by connecting a rheostat across the solar panel. When the resistance across the solar panel is 0 , the solar panel is short-circuited, therefore, voltage $V_{S C}=0$ and the short-circuit current $I_{S C}$ is found. When the both the ends of solar panel are disconnected from rheostat, we get an open circuit condition where, $I_{O C}=0$ and $V_{O C}$ is maximum. Remaining values of voltage and current of solar panels are found by gradually increasing the resistance from 0 to $\infty$ The above approach is repeated for different values of solar intensity under controlled illumination in steps of $200 \frac{\mathrm{W}}{\mathrm{m}^{2}}$. The results plotted in Fig. 5 suggests that the V-I characteristics derived from analytical model (plotted using dashed line) matches closely with experimental results (plotted using solid line).

We conduct numerical investigation for the proposed models by using the default system parameters mentioned next. We consider a $3 \mathrm{~W}$ solar panel whose parameters are listed in Table I. Unless otherwise specified a $40 \mathrm{~F}$ supercapacitor is considered with a residual voltage $V_{\text {res }}$ equal to minimum threshold voltage $V_{l}=3.3 \mathrm{~V}$. In order to account for the spatial variation of solar intensity $G$, we consider 2 different geographic locations, namely, New Delhi, and Quebec city. The long term average solar intensity received during sunlight time at these places are respectively $428 \mathrm{~W} / \mathrm{m}^{2}$, and $303 \mathrm{~W} / \mathrm{m}^{2}$. We consider Libelium Waspmote [27] as the sensor node, which is connected in parallel to the supercapacitor as shown in Fig. 1. Maximum of 6 gas sensors (carbon monoxide $(\mathrm{CO})$, ammonia $\left(\mathrm{NH}_{3}\right)$, nitrogen dioxide $\left(\mathrm{NO}_{2}\right)$, carbon dioxide $\left(\mathrm{CO}_{2}\right)$, volatile organic compounds $(\mathrm{VOC})$, and methane $\left(\mathrm{CH}_{4}\right)$ ) can be mounted on-board waspmote. For calculating the average power consumption $P_{\text {cons }}^{a v g}$ we adopt the approach in [40] where consumptions due to all operation states, namely, sense, datalog, sleep and communication, were considered. The consumption of all the individual modules in the waspmote are provided in Table II. From [27] we get the size of data per sample as $21+4 \times N_{s n}$ bytes. Further, using [41], [27] we get the overhead and the maximum packet size respectively for communication as 18 and 92 bytes.

\section{A. Validation of Analysis}

First of all, we validate the analytically obtained results for the charging rate $\mathrm{CDF} F_{\Gamma}$ and energy outage probability via simulations. For simulation of $F_{\Gamma}$ with the data set of New Delhi area, the solar intensity values obtained from NREL are substituted in (3a). The solar current $I_{i r r}$ is substituted in (11) to get $\Gamma_{a}$. This is used to obtain the simulated CDF of solar charging rate $F_{\Gamma_{a}}$ for approximate solar panel model.
TABLE II: Voltage and Current consumption of Waspmote [27] components.

\begin{tabular}{lrrr}
\hline Component & Voltage $(\mathrm{V})$ & Current $(\mathrm{mA})$ & Time $(\mathrm{s})$ \\
\hline Gas sensor & & & \\
$\mathrm{CO}$ & 5 & 3 & 1 \\
$\mathrm{NH}_{3}$ & 5 & 12 & 0.25 \\
$\mathrm{NO}_{2}$ & 1.8 & 26 & 30 \\
$\mathrm{VOC} \mathrm{CO}_{2}$ & 2.5 & 32 & 30 \\
$\mathrm{CH}_{4}$ & 5 & 50 & 90 \\
Waspmote & 5 & 61 & 30 \\
Active & & & \\
Sleep & 3.7 & 15 & \\
Sensor board & 3.7 & $180 \times 10^{-3}$ & \\
MicroSD card & 3.3 & 2 & \\
On & & & \\
Write 1 byte & 3.3 & 0.14 & \\
Read 1 byte & 3.3 & 0.2 & $1.75 \times 10^{-3}$ \\
Radio & 3.3 & 0.2 & $1.75 \times 10^{-3}$ \\
On & & & \\
Transmit 1 byte & 3.3 & 37.38 & \\
Receive 1 byte & 3.3 & 37.98 & $31.25 \times 10^{-6}$ \\
\hline
\end{tabular}

Analytical CDF of solar charging rate $F_{\Gamma_{a}}$ is obtained using the closed-form expression for $F_{\Gamma_{a}}$ in (17b). We plotted $F_{\Gamma_{a}}$ for both polynomial-fit based mixture distribution of $G$ (using (16)) against the numerical simulation in Fig. 6. A similar method is followed to plot $F_{\Gamma_{p}}$ for practical solar panel model using (5), (18a), and (18b) in Fig. 6. We have repeated this whole exercise for Quebec city area and plotted the results.

Fig. 6 shows that for both the locations, $F_{\Gamma_{a}}$ and $F_{\Gamma_{p}}$ found using polynomial-fit based mixture distribution matches closely with the simulation result. Moreover, the results obtained for a particular location via both solar panel models match. This is due to the fact that both solar panel models behave in a similar fashion between the voltages of our interest, i.e., $V_{\min }=3.3 \mathrm{~V}$ and $V_{\max }=3.8 \mathrm{~V}$ (cf. Fig. 3). It can be noted from Fig. 6 that, for any CDF value, New Delhi area has the higher solar charging rate due to its higher average solar intensity compared to the Quebec city area.

As energy outage probability $p_{\text {out }}$ is itself a function of $F_{\Gamma}$, providing verification for $F_{\Gamma}$ in turn provides the $p_{\text {out }}$ validation.

\section{B. Trade-off between Energy Outage Probability $p_{\text {out }}$ and Sampling Rate $r_{s}$}

At both the locations, a Waspmote with either $1(C O)$ or all 6 on-board gas sensors and a $40 \mathrm{~F}$ supercapacitor are considered. For various values of $p_{\text {out }}$, in Fig. 7 we have plotted $r_{s}$ obtained from equations (20c) and (1). $r_{s}$ obtained at each place for approximate model matches closely with that of practical model of solar panel. Note that beginning from the outage probabilities of 0.29 and 0.65 respectively for 1 sensor and 6 sensors, the sampling rates become constant. This is due to fact that each gas sensor has a minimum response time and the samples taken with sampling rates greater than it results in the sensor producing inaccurate results. 


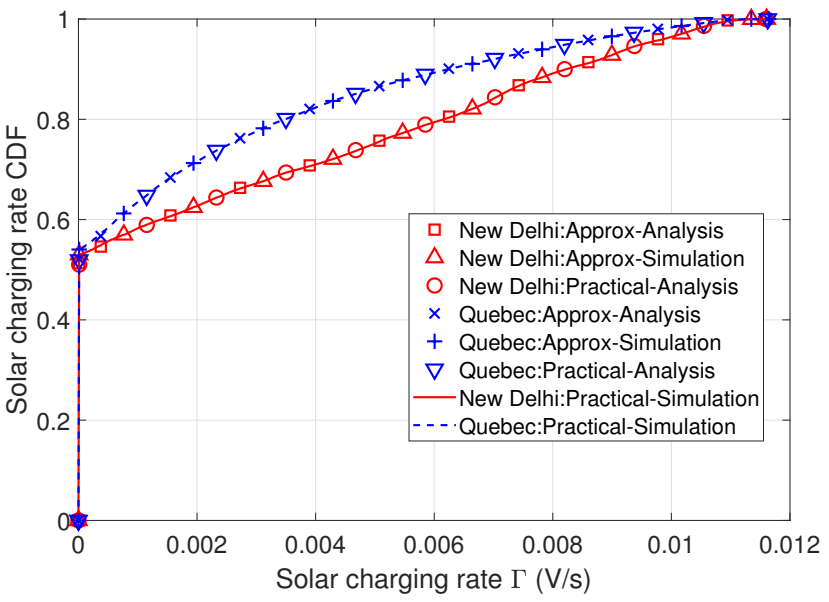

Fig. 6: Validation of solar charging rate CDF for different regions.

\section{Results on Sizing the Components}

1) Optimal Solar Panel Size: Considering diode $D_{1}$ with cut-off voltage $V_{x}^{\text {ext }}=0.15 \mathrm{~V}$ and using the reference parameters in Table I, we have calculated the number of solar cells $N_{S}$ for both approximate and practical solar panel models needed at both locations using the conditions mentioned in Section VI-C. For computation of $N_{S}^{a}$ using approximate solar panel model, we have assumed $\Delta I=1 \mathrm{~mA}$. The obtained non-interger values for either of $N_{S}^{a}$ or $N_{S}^{p}$, are rounded to the next integer. As mentioned in Table III, we get $N_{S}^{p}=7$ and $N_{S}^{a}=11$ for both places. Since a higher value of $N_{S}^{p}$ allows the supercapacitor to be charged to a higher voltages $V_{C}>V_{\max }$, diode $D_{1}$ with a higher cut-off voltage $V_{x}^{\text {ext }}$ should be used instead of the zener diode such that the supercapacitor is only charged up to $V_{\max }$.

2) Optimal Supercapacitor Size: The solar panel size $N_{S}^{a}$ or $N_{S}^{p}$ computed above is used as the input in order to calculate supercapacitor size $C_{1}$, which is obtained using Algorithm 1 for the approximate solar panel model. For practical solar panel model, $C_{1}^{l}$ and $C_{1}^{u}$ are obatined using Algorithm 2.

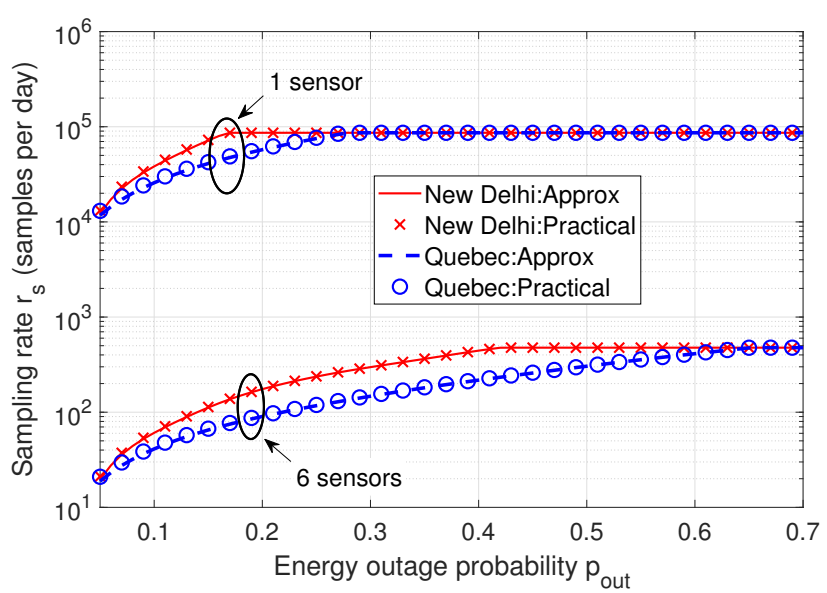

Fig. 7: Sampling rate versus energy outage probability.
We also computed supercapacitor size $C_{2}$ using (26). The supercapacitor sizes computed for both places by assuming different number of sensors and sampling intervals are shown in Table III. While $N_{s n}=1$ corresponds to a single $C O$ sensor, $N_{s n}=6$ indicates that all 6 gas sensors are on-board waspmote.

Since $C_{1}^{u}$ is the supercapacitor size when the node just harvests all the available solar energy, i.e., $P_{\text {cons }}^{a v g}=0$, it is unique which depends just on the location. For both solar panel models at a particular location, $C_{1}$ is the highest when $N_{s n}=1$ and sampling interval $=60 \mathrm{~min}$, and lowest when $N_{s n}=6$ with sampling interval $=30 \mathrm{~min}$. This is since $C_{1}$ is inversely proportional to the average power consumption of sensor node. The supercapacitor values of $C_{1}, C_{1}^{l}$ and $C_{1}^{u}$ are much higher compared $270 \mathrm{~F}$, which is the maximum supercapacitor value that can be charged till $3.8 \mathrm{~V}$ available in the market [39]. To this end, to realize the required high value, the commercially available supercapacitors has to be connected in parallel. The optimal value of the supercapacitor needed to support a particular QoS (decided by $N_{s n}$ and sampling interval) is in the range $\left[C_{2}, C_{1}\right]$, which can be decided based on location of the place and the available budget.

Lastly, we would like to mention that the solar energy harvesting wireless sensor node with a supercapacitor size $C_{1}$ and solar panel size $N_{s}^{a}$ as mentioned in the Table II is able to provide zero energy outage probability when the residual energy at the supercapacitor at the initial time is greater than or equal to energy $E_{\min }+\Delta E$. Here, $E_{\min }$ is the energy of the supercapacitor at $V_{\min }=3.3 \mathrm{~V}$ and $\Delta E$ is the additional energy required as mentioned in last column of Table III. This energy $\Delta E$ ensures that the mentioned sustainable sampling rate can be achieved with zero outage probability.

\section{Discussion on Use Cases}

1) Practical Solar Panel Model : Practical solar panel model can be used when the load is directly connected across the supercapacitor as the number $N_{S}$ of solar cells connected in this way ensures that load voltage stays in its working range [19]. As a result, the practical solar panel model can be used for WSN deployments both indoors [15] and outdoors [19] without any circuit for monitoring the supercapacitor voltage.

2) Approximate Solar Panel Model : Approximate solar panel model should be used when fast charging is required on an average. Fast charging is achieved by ensuring that the charging current is linear on average by restricting the working range of the solar panel. However, we need a supercapacitor monitoring and control circuit, which switches off the charging path between solar panel and supercapacitor when supercapacitor charges to $V_{\max }$, and switches on when the supercapacitor voltage drops down below $V_{\text {max }}$.

In Fig. 3 we demonstrated that at certain solar intensities, approximate solar model matches closely with practical solar panel model. However, this is not true when solar intensity $G$ is low (observed indoors) since the parallel resistance $R_{P}$ (which is proportional to $G$ ) acts as a low resistance path for 
TABLE III: Solar panel and supercapacitor sizes.

\begin{tabular}{|c|c|c|c|c|c|c|c|c|c|}
\hline \multirow{2}{*}{ Location } & \multirow{2}{*}{$\mathbf{N}_{\mathrm{sn}}$} & \multirow{2}{*}{ Sampling Interval (min) } & \multirow{2}{*}{$\mathrm{C}_{2}(\mathrm{~F})$} & \multicolumn{3}{|c|}{ Practical } & \multicolumn{3}{|c|}{ Approximate } \\
\hline & & & & $\mathbf{N}_{\mathrm{S}}^{\mathrm{P}}$ & $\mathrm{C}_{1}^{\mathrm{l}}(\mathrm{F})$ & $\mathrm{C}_{1}^{\mathrm{u}}(\mathrm{F})$ & $\mathbf{N}_{\mathrm{S}}^{\mathrm{a}}$ & $\mathrm{C}_{1}(\mathrm{~F})$ & $\Delta \mathrm{E}(\mathbf{m} \mathbf{J})$ \\
\hline \multirow{4}{*}{$\begin{array}{l}\text { New } \\
\text { Delhi }\end{array}$} & 1 & 30 & 17.3 & \multirow{4}{*}{7} & 11508 & \multirow{4}{*}{17325} & \multirow{4}{*}{11} & 17325 & 1.440 \\
\hline & 1 & 60 & 16.7 & & 11580 & & & 17326 & 1.396 \\
\hline & 6 & 30 & 664.4 & & 2059 & & & 16673 & 55.439 \\
\hline & 6 & 60 & 340.3 & & 3363 & & & 17000 & 28.396 \\
\hline \multirow{4}{*}{$\begin{array}{l}\text { Quebec } \\
\text { city }\end{array}$} & 1 & 30 & 18 & \multirow{4}{*}{7} & 6029 & \multirow{4}{*}{11735} & \multirow{4}{*}{11} & 12251 & 1.473 \\
\hline & 1 & 60 & 17.4 & & 6089 & & & 12252 & 1.429 \\
\hline & 6 & 30 & 692.1 & & 597 & & & 11599 & 56.732 \\
\hline & 6 & 60 & 354.5 & & 1069 & & & 11926 & 29.059 \\
\hline
\end{tabular}

the generated solar current $I_{i r r}$. Therefore, in order to stay in the linear operation region, a higher number of solar panels $N_{S}$ would be required.

\section{CONCLUding REMARKS}

In this work we have studied the sustainability of WSN nodes powered by solar panels. We have obtained closed-form expressions for solar charging rate distribution and energy outage probability for both approximate and practical solar panel models proposed in this paper. Extensive numerical investigation have been conducted to validate accuracy of the developed models and provide insights on the performance of sensor node with varying practical system parameters, such as, sensing rate and number of sensors on the node. Additionally, optimal sizes of supercapacitor and solar panel for sustained the node operation under a given solar intensity distribution and average power consumption of the node have been derived using the expected solar charging rate.

The analysis presented in this paper helps to identify the necessary resources for sustainable operation of an energy harvesting wireless sensor node even before deployment at a particular location. In this analysis a mobile entity-based data collection is considered. However, it is not seen if the cost of the whole network could be further brought down by considering a large solar panel at the mobile entity and RF energy harvester at the nodes. Since the mobile entity can move around the field, it can put itself in a position where a significant amount of energy can be harvested at any given time. We plan to move in this direction in the future.

\section{REFERENCES}

[1] Akyildiz, I.F., Melodia, T., Chowdhury, K.R.: 'Wireless multimedia sensor networks: Applications and testbeds', Proc IEEE, 2008, 96, (10), pp. 1588-1605

[2] He, Y., Cheng, X., Peng, W., Stuber, G.L.: 'A survey of energy harvesting communications: models and offline optimal policies', IEEE Commun Mag, 2015, 53, (6), pp. 79-85

[3] Jeong, J., Culler, D.: 'A practical theory of micro-solar power sensor networks', ACM Trans Sen Netw, 2012, 9, (1), pp. 9:1-9:36

[4] Cammarano, A., Petrioli, C., Spenza, D.: 'Online energy harvesting prediction in environmentally powered wireless sensor networks', IEEE Sensors J, 2016, 16, (17), pp. 6793-6804

[5] Escolar, S., Chessa, S., Carretero, J.: 'Energy management in solar cells powered wireless sensor networks for quality of service optimization', Personal and Ubiquitous Computing, 2014, 18, (2), pp. 449-464

[6] 'Libelium waspmote - case studies'. (, . accessed: Dec. 12, 2018. Available from: http://www.libelium.com/libeliumworld/case-studies/

[7] Lee, S., Lee, I., Kim, S., Lee, S., Bovik, A.C.: 'A pervasive network control algorithm for multicamera networks', IEEE Sensors J, 2014, 14, (4), pp. 1280-1294
[8] Yang, J., Ulukus, S.: 'Optimal packet scheduling in an energy harvesting communication system', IEEE Trans Commun, 2012, 60, (1), pp. 220230

[9] Ozel, O., Tutuncuoglu, K., Yang, J., Ulukus, S., Yener, A.: 'Transmission with energy harvesting nodes in fading wireless channels: Optimal policies', IEEE J Sel Areas Commun, 2011, 29, (8), pp. 1732-1743

[10] Yang, J., Ozel, O., Ulukus, S.: 'Broadcasting with an energy harvesting rechargeable transmitter', IEEE Trans Wireless Commun, 2012, 11, (2), pp. 571-583

[11] Sharma, V., Mukherji, U., Joseph, V., Gupta, S.: 'Optimal energy management policies for energy harvesting sensor nodes', IEEE Trans Wireless Commun, 2010, 9, (4), pp. 1326-1336

[12] Tian, H., Mancilla.David, F., Ellis, K., Muljadi, E., Jenkins, P.: 'A cell-tomodule-to-array detailed model for photovoltaic panels', Solar Energy, 2012, 86, (9), pp. $2695-2706$

[13] Ishaque, K., Salam, Z., Taheri, H.: 'Simple, fast and accurate two-diode model for photovoltaic modules', Solar Energy Materials and Solar Cells, 2011, 95, (2), pp. 586 - 594

[14] 'Case studies of practical wireless sensor networks deployment'. (, . accessed: Apr. 22, 2017. Available from: http://www.libelium.com/ resources/case-studies/

[15] Gorlatova, M., Wallwater, A., Zussman, G.: 'Networking low-power energy harvesting devices: Measurements and algorithms', IEEE Trans Mobile Comput, 2013, 12, (9), pp. 1853-1865

[16] Heinzelman, W.R., Chandrakasan, A., Balakrishnan, H. 'Energy-efficient communication protocol for wireless microsensor networks'. In: Proc. 33rd Annual Hawaii Int. Conf. System Sciences. (Hawaii, USA, 2000. pp. 10 pp. vol.2-

[17] Deng, F., Yue, X., Yue, X., Fan, X., Guan, S., Xu, Y., et al.: 'Multisource energy harvesting system for a wireless sensor network node in the field environment', IEEE IoT J, 2018, pp. 1-1

[18] Corke, P., Valencia, P., Sikka, P., Wark, T., Overs, L. 'Long-duration solar-powered wireless sensor networks'. In: Proc. 4th Workshop on Embedded Networked Sensors. (New York, USA, 2007. pp. 33-37

[19] Raghunathan, V., Kansal, A., Hsu, J., Friedman, J., Srivastava, M. 'Design considerations for solar energy harvesting wireless embedded systems'. In: Proc. IPSN. (Los Angeles, USA, 2005. pp. 457-462

[20] Taneja, J., Jeong, J., Culler, D. 'Design, modeling, and capacity planning for micro-solar power sensor networks'. In: Proc. IPSN. (St. Louis, USA, 2008. pp. 407-418

[21] Jiang, X., Polastre, J., Culler, D. 'Perpetual environmentally powered sensor networks'. In: Proc. IPSN. (Los Angeles, USA, 2005. pp. 463 468

[22] Dutta, P., Hui, J., Jeong, J., Kim, S., Sharp, C., Taneja, J., et al. 'Trio: enabling sustainable and scalable outdoor wireless sensor network deployments'. In: Proc. IPSN. (Nashville, USA, 2006. pp. 407-415

[23] Musiani, D., Lin, K., Rosing, T.S. 'Active sensing platform for wireless structural health monitoring'. In: Proc. IPSN. (Cambridge, USA, 2007. pp. 390-399

[24] Park, C., Chou, P.H. 'Ambimax: Autonomous energy harvesting platform for multi-supply wireless sensor nodes'. In: Proc. IEEE SECON. vol. 1. (Reston, USA, 2006. pp. 168-177

[25] Simjee, F., Chou, P.H. 'Everlast: Long-life, supercapacitor-operated wireless sensor node'. In: Proc. ISLPED. (Tegernsee, Germany, 2006. pp. 197-202

[26] Minami, M., Morito, T., Morikawa, H., Aoyama, T. 'Solar biscuit: A battery-less wireless sensor network system for environmental monitoring applications'. In: Proc. 2nd Int. Workshop Netw. Sens. Syst.,. (San Diego, USA, 2005. pp. 1-6 
[27] 'Libelium waspmote'. (, . accessed: Apr. 22, 2017. Available from: http://www.libelium.com/products/waspmote/

[28] Kansal, A., Hsu, J., Zahedi, S., Srivastava, M.B.: 'Power management in energy harvesting sensor networks', ACM Trans Embed Comput Syst, 2007, 6, (4)

[29] Cid.Fuentes, R.G., Cabellos, A., Alarcon, E. 'Energy harvesting enabled wireless sensor networks: Energy model and battery dimensioning'. In: Proc. 7th Int. Conf. on Body Area Networks. (Oslo, Norway, 2012. pp. 131-134

[30] Chamola, V., Sikdar, B.: 'A multistate markov model for dimensioning solar powered cellular base stations', IEEE Trans Sustain Energy, 2015, 6, (4), pp. 1650-1652

[31] Jelicic, V., Magno, M., Brunelli, D., Bilas, V., Benini, L. 'Analytic comparison of wake-up receivers for wsns and benefits over the wakeon radio scheme'. In: Proc. 7th ACM Workshop on Performance Monitoring and Measurement of Heterogeneous Wireless and Wired Networks. (New York, USA, 2012. pp. 99-106

[32] Kaushik, K., Mishra, D., De, S., Chowdhury, K.R., Heinzelman, W. 'Low-cost wake-up receiver for RF energy harvesting wireless sensor networks', IEEE Sensors J, 2016, 16, (16), pp. 6270-6278

[33] Mishra, D., De, S.: 'Effects of practical rechargeability constraints on perpetual RF harvesting sensor network operation', IEEE Access, 2016, 4, pp. 750-765

[34] Jeong, J., Jiang, X., Culler, D. 'Design and analysis of micro-solar power systems for wireless sensor networks'. In: Proc. 5th Int. Conf. on Networked Sensing Systems. (Kanazawa, Japan, 2008. pp. 181-188

[35] Rajapakse, A.D., Muthumuni, D. 'Simulation tools for photovoltaic system grid integration studies'. In: Proc. IEEE Electr. Power Energy Conf. (Montreal, Canada, 2009. pp. 1-5

[36] Mishra, D., De, S., Chowdhury, K.R.: 'Charging time characterization for wireless RF energy transfer', IEEE Trans Circuits Syst II, Exp Briefs, 2015, 62, (4), pp. 362-366

[37] Boylestad, R.L., Nashelsky, L.: 'Electronic Devices and Circuit Theory (6th Ed.)'. (Upper Saddle River, NJ, USA: Prentice-Hall, Inc., 1996)

[38] 'National renewable energy labaratory database'. (, . accessed: Apr. 22, 2017. Available from: https://nsrdb.nrel.gov/nsrdb-viewer

[39] 'Mouser electronics'. (, . accessed: Apr. 27, 2017. Available from: http://www.mouser.in/

[40] Gupta, P., Kandakatla, K., De, S., Jana, S. 'Feasibility analysis on integrated recharging and data collection in pollution sensor networks'. In: Proc. NCC. (New Delhi, India, 2013. pp. 1-5

[41] 'Digi international xbee zigbee module'. (, . accessed: Apr. 22, 2017. Available from: https://www.digi.com/products/xbee-rf-solutions/ embedded-rf-modules-modems/digi-xbee-zigbee 